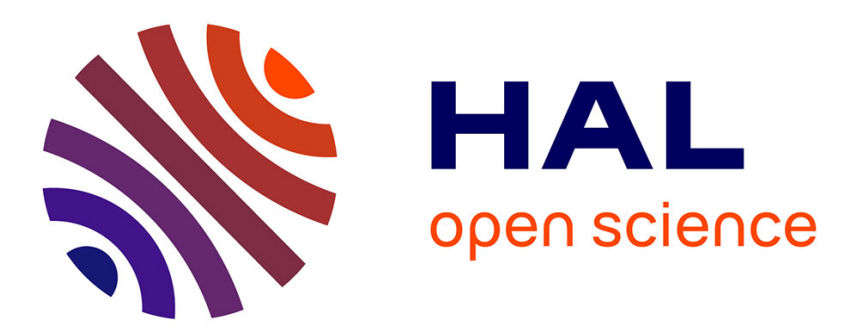

\title{
Mutualité et capitalisme entre 1789 et 1947 : de la subversion à l'intégration
}

Nicolas da Silva

\section{To cite this version:}

Nicolas da Silva. Mutualité et capitalisme entre 1789 et 1947: de la subversion à l'intégration. Revue Internationale de l'Economie Sociale, 2020, 357. hal-03228414

\section{HAL Id: hal-03228414 \\ https://hal.science/hal-03228414}

Submitted on 18 May 2021

HAL is a multi-disciplinary open access archive for the deposit and dissemination of scientific research documents, whether they are published or not. The documents may come from teaching and research institutions in France or abroad, or from public or private research centers.
L'archive ouverte pluridisciplinaire HAL, est destinée au dépôt et à la diffusion de documents scientifiques de niveau recherche, publiés ou non, émanant des établissements d'enseignement et de recherche français ou étrangers, des laboratoires publics ou privés. 


\title{
Mutualité et capitalisme entre 1789 et 1947 : de la subversion à l'intégration
}

\author{
Nicolas Da Silva, Université Paris 13, CEPN UMR 7234
}

\begin{abstract}
Résumé
Cet article propose une relecture de l'histoire économique de la mutualité entre 1789 et 1947 au prisme de son rapport au capitalisme. Alors qu'après la Révolution française et jusqu'à la Commune de Paris la mutualité est une institution ambiguë, entre subversion et intégration au capitalisme, à partir de 1871 elle s'insère largement au capitalisme. C'est à cette période que se séparent mutualisme et syndicalisme. Initialement, les classes populaires sont contraintes de s'auto-organiser pour survivre au dépérissement des protections de l'Ancien Régime et au libéralisme asymétrique de la bourgeoisie. Toute la finesse de la réglementation de l'État sous le Second Empire et de la IIIème République est d'essayer d'intégrer la mutualité pour en vider le contenu contestataire. Contrairement aux interprétations classiques, on peut alors dire que la Sécurité sociale de 1945 n'est pas la revanche des syndicalistes sur les mutualistes mais celle d'une certaine forme de la mutualité contre une autre.
\end{abstract}

\section{Introduction}

L'un des aspects majeurs du débat actuel sur le financement de la santé en France porte sur la place respective qu'il convient d'attribuer à la Sécurité sociale et aux complémentaires (mutuelles, institutions de prévoyance et sociétés d'assurance). Étonnamment, ce débat n'interroge pas les raisons historiques de la séparation en France entre la Sécurité sociale et les complémentaires. Afin d'éclairer les discussions contemporaines nous proposons ici une relecture de l'histoire économique et politique de la mutualité entre 1789 et $1947^{1}$. L'objectif de cet article est d'étudier les différentes formes de la mutualité depuis 1789 pour comprendre les relations conflictuelles entre la mutualité et la Sécurité sociale. La mutualité est indissociable de l'histoire de la lutte des classes et du développement du capitalisme en France. Elle s'inscrit dans le cadre du conflit capital-travail et propose des solutions variées au cours de son histoire. La thèse développée ici est qu'après une période durant laquelle la mutualité fut une institution ambivalente au regard du capitalisme - entre la subversion de l'ordre établi et son acceptation, elle est devenue une institution accompagnatrice des évolutions du capitalisme. Ainsi, la Sécurité sociale de 1945 n'est pas une institution en opposition à la mutualité mais en opposition à une forme historique de la mutualité - la mutualité d'Empire et la mutualité de la IIIème République. Le rôle de l'État dans ce mouvement historique n'est pas neutre : à partir de 1852 il a cherché à se réapproprier l'institution pour en favoriser une dimension plutôt qu'une autre: l'accommodation au capitalisme plutôt que sa critique.

Le texte se compose de quatre sections. Dans la première section, nous replaçons la mutualité dans le contexte du XIXème siècle qui met les classes populaires dans un étau entre le libéralisme asymétrique de la Révolution française et la misère de l'industrialisation. La mutualité est alors un objet ambivalent, entre conservation et remise en cause de l'ordre établi. La seconde section porte sur la mutualité après le décret de 1852 par lequel l'État cherche à se réapproprier les sociétés de secours mutuels pour en détourner l'esprit démocratique. La troisième section s'intéresse à la période républicaine de la mutualité qui

\footnotetext{
${ }^{1}$ Ce travail s'inscrit dans le cadre de l'ANR « Marché du risque santé : construction, gouvernance, innovation » (oct. 2017-oct. 2020).
} 
voit cette dernière basculer lentement vers l'acceptation complète de l'ordre établi tandis que la branche syndicaliste du mouvement ouvrier prend le flambeau de la contestation. La quatrième section interprète la naissance de la Sécurité sociale non comme la revanche des syndicalistes sur les mutualistes mais comme l'avènement d'une forme de la mutualité contre une autre. La Sécurité sociale met en pratique les aspirations de la mutualité subversive de l'ordre établi.

\section{Révolutions et interdiction de la mutualité (1789-1852)}

La signification économique et politique de l'institution mutualiste ne s'éclaire que si l'on rappelle le contexte révolutionnaire de la période ouverte à la fin du XVIII ${ }^{\text {ème }}$ siècle. Dans son histoire du «long XIX $^{\text {ème }}$ siècle »(1789-1914), Éric Hobsbawm souligne à quel point la double révolution de 1780-1848 modifie durablement les rapports sociaux en France, en Europe et dans les pays sous influence européenne (Hobsbawm, 1988). S'il est toujours possible de mettre en évidence des continuités fortes, la Révolution industrielle et la Révolution française rompent radicalement avec les sociétés de l'Ancien Régime. Ces ruptures s'inscrivent dans le passage du féodalisme au capitalisme et expliquent la centralité de la mutualité.

La grande originalité de la Révolution française par rapport aux révolution précédentes est qu'elle est une révolution de masse, impliquant les classes populaires, et non une révolution d'élites (Skocpol, 1979). Alors qu'en Angleterre la bourgeoise et l'aristocratie parviennent à un compromis politique sur la répartition du pouvoir, en France, l'aristocratie a refusé ce compromis obligeant la bourgeoisie française à mobiliser derrière elle les travailleurs ruraux et urbains (Kates, 2006). Si l'on garde souvent, à juste titre, la mémoire d'une Révolution française très violente, la justification de cette violence reste relativement méconnue : la destruction des institutions féodales.

Il faut souligner deux caractéristiques principales de la Révolution française pour retracer l'histoire de la mutualité. D'une part, la Révolution française débouche sur un libéralisme économique débridé, le laissez-faire, laissez-passer. En plus de l'abolition des privilèges féodaux, les protections de l'Ancien Régime sont dissoutes (corporations, douanes intérieures et extérieures, etc.) dans le but de construire un marché national homogène où peut s'exercer la concurrence. La défense de la propriété et de la libre entreprise va même jusqu'à interdire toute forme d'organisation de travailleurs destinée à défendre leurs intérêts professionnels (lois d'Allarde et le Chapelier de 1791), ce qui rend illégal le syndicalisme ou même la constitution de mutuelles. Cela ne signifie pas qu'il n'y pas une régulation juridique du travail : les capitalistes s'exonèrent de leur responsabilité vis-à-vis du travail par l'institution du contrat de louage d'ouvrage (Didry, 2016).

D'autre part, la Révolution française produit un libéralisme politique inégalitaire. La constitution de 1791 est exemplaire du désir majoritaire de la bourgeoisie française. Elle souhaite accéder au pouvoir politique sans pour autant remettre en cause la monarchie et tout en refusant l'accès au pouvoir aux masses populaires. Cette constitution prévoit un suffrage censitaire qui n'accorde le statut de citoyen actif qu'à ceux qui peuvent l'acheter. L'enjeu est clair : il faut écarter du pouvoir les individus susceptibles de remettre en cause la propriété. Dans cet esprit, les constituants préfèrent un régime représentatif (de délégation de pouvoir) à un régime démocratique (où le pouvoir serait exercé directement par les citoyens). Les événements de l'An II donnent raison à la bourgeoisie dans sa crainte de voir la propriété menacée par un régime trop démocratique. Les principes de liberté et d'égalité sont un problème dès lors qu'ils menacent les intérêts de la bourgeoisie (Margairaz, 1991). 
Parallèlement à la Révolution française, la période 1780-1848 est celle de la Révolution industrielle. Celle-ci se déploie plus tardivement et plus lentement en France qu'en Angleterre mais ses effets sont tout aussi déstructurants. La production dans les secteurs moteurs de l'industrialisation s'accélère (textile, chemin de fer, mines, etc.) en renforçant la séparation entre le travailleur et les moyens de production. L'industrialisation engendre une urbanisation rapide et désorganisée. L'agriculture tend à se concentrer, même si la France se caractérise par la prégnance de petites et moyennes propriétés.

Ces changements dans la production ont un impact direct sur les travailleurs. Les conditions de travail sont dégradées par rapport aux activités rurales et artisanales traditionnelles (modification des cadences, accidents du travail plus réguliers et plus graves, extension de la durée de travail, etc.). L'urbanisation mal contrôlée pose des problèmes d'hygiène publique, ce qui constitue un terreau favorable pour les épidémies. Les contemporains René-Louis Villermé pour la France et Friedrich Engels pour l'Angleterre ont brossé en ce sens un tableau édifiant de la situation de la classe ouvrière.

Au sortir de cette double révolution, les classes populaires sont prises en étau entre la brutalité de l'économie libérale et le refus obstiné de l'État de remplacer les institutions de l'Ancien Régime autrefois chargées de prendre soin des plus démunis. Sous l'Ancien Régime, le clergé organisait la charité (notamment en entretenant les hôpitaux) et les communautés (villageoise et familiale) constituaient un ultime rempart face à la misère. Désormais, l'Église, ne percevant plus la dîme, n'est plus en position de faire la charité et l'urbanisation bouleverse profondément les solidarités traditionnelles. Le seul acteur capable de prendre le relais des institutions féodales est l'État, mais celui-ci se réfugie derrière ce que le sociologue Henri Hatzfeld (1971) a appelé “l'objection libérale". L'État ne doit pas intervenir car le remède serait pire que le mal (incitation à la paresse et à la débauche, remise en cause de la propriété, transformation de la vertueuse charité en contrat, etc.).

Le refus de l'intervention de l'État et la violence des rapports capitalistes imposent une méthode aux travailleurs urbains et ruraux qui veulent améliorer leur sort: 1'autoorganisation, contre l'État et contre le capital. Les sociétés de secours mutuels s'inscrivent dans cette histoire. Alors qu'en Angleterre les friendly societies sont rapidement légalisées, les mutuelles en France sont beaucoup plus ambivalentes dans leur rapport à l'État. Elles sont interdites lorsqu'on les soupçonne de tendre vers une forme de coalition ouvrière, mais elles sont tolérées tant qu'elles ne remettent pas en cause l'ordre établi au sortir de la Révolution de 1789.

À l'aube de la Révolution de 1848, les sociétés de secours mutuels comptent 2500 entités pour 270000 membres (Daviet, 1999). Pendant la période 1789-1852 elles sont à mi-chemin entre la survivance des formes de sociabilité de l'Ancien Régime (compagnonnage, confrérie, corporation, etc.) et le renouveau des débuts du Second empire. Cette ambivalence explique la tolérance vis-à-vis de la mutualité. Pour l'État (Premier Empire ou Restauration), la mutualité est considérée comme une organisation collective stabilisante rappelant la société d'ordres de l'Ancien régime. Elles sont également un moyen de soulager les finances de l'empire dont les hôpitaux et les bureaux de bienfaisance ne peuvent faire face à l'augmentation de la demande de services sociaux (Weintrob, 1999). Il arrive même que les préfets soient à l'initiative de la création de sociétés de secours mutuels dans le but prendre en charge les accidents du travail sinon propices à l'agitation. Les notables voient dans la mutualité une institution susceptible de stabiliser les relations sociales en modifiant le comportement des classes populaires.

Ces aspirations n'en rencontrent pas moins l'hostilité chez certains ouvriers. Si les activités principales des mutuelles sont bien celles qui expliquent la tolérance du pouvoir à leur égard 
(organisation de fêtes, visites aux malades, secours aux anciens, financement des frais funéraires), les mutuelles sont aussi un lieu de contestation qui souffre de la répression. Elles sont utilisées comme des «comités des lutte» (Daviet, 1999, p.45): surveillance et négociations des conditions de travail, discussion des tarifs pour le travail salarié, organisation des luttes, etc.). Les mutualités sont enfin le lieux d'apprentissage des codes et pratiques de la démocratie et de la citoyenneté à une période où les classes populaires sont explicitement maintenues dans une position de minorité politique.

La Révolution de 1848 ressemble à s'y méprendre à celle de 1789 : la bourgeoisie fait appel aux masses populaires pour conquérir le pouvoir et elle maintient sa préférence pour la propriété face à la démocratie en basculant rapidement vers le Second Empire. Alors que la Constitution de 1848 proclamait la liberté d'association, dès juin 1849 de nombreuses associations sont dissoutes au nom du respect de l'ordre public, c'est-à-dire de l'ordre bourgeois.

\section{La mutuelle d'empire : entre subversion et réappropriation (1852-1871)}

Dans un contexte d'accélération de l'industrialisation et de hausse de la population ouvrière, le Second empire amorce un changement de stratégie de la part de l'État vis-à-vis de la question mutualiste. La libéralisation temporaire de 1848 a été un échec. Mais, pour le pouvoir le retour à l'ambivalence de la période précédente n'est pas satisfaisant. Plutôt que d'utiliser uniquement la répression, l'État va chercher à se réapproprier l'institution mutualiste par la création d'un cadre législatif et économique incitatif. L'objectif est de transformer les mutuelles : d'institutions ouvrières potentiellement subversives du capitalisme (organisation d'une conflictualité de classe, auto-organisation, etc.), elles doivent devenir des forces intégrées au capitalisme (organiser le secours contre les risques liés au travail industriel, éviter l'agitation ouvrière, etc.). Cette stratégie de l'État conduit progressivement à la scission entre le mutuellisme (pacifié) et le syndicalisme (contestataire).

Le décret du 26 mars 1852 créé un cadre légal pour les mutuelles distinguant strictement les mutuelles approuvées et les mutuelles autorisées. Les mutuelles approuvées bénéficient de nombreux avantages en contrepartie d'un contrôle politique fort des élites de l'Empire tandis que les mutuelles autorisées ne bénéficient d'aucun avantage et attirent vers elles les forces de la répression par leur refus de l'approbation. Ce décret créé une forme d'auto-sélection entre les mutuelles acceptant l'ordre établi et celles qui souhaitent conserver leur autonomie.

Les mutuelles approuvées subissent un contrôle politique massif en échange de nombreux avantages (Toucas-Truyen, 1999). Traditionnellement liées aux métiers, les mutuelles sont principalement organisées sur une base territoriale afin de limiter la coordination des professions (sur 236 création de mutuelles en 1852, seules 21 le sont sur base corporatiste). Elles sont hiérarchiquement placées sous l'autorité du maire (nommé par les préfectures) puis du préfet (qui peut décider de la dissolution) et de la Commission supérieure d'encouragement et du surveillance (qui dépend du ministère de l'Intérieur). Contrairement aux mutuelles autorisées qui peuvent élire librement leurs présidents, les présidents des mutuelles approuvées sont choisis par l'État. Or, le «comportement politique arrive au premier rang des critères de sélection » (ibid. p.16). Par ailleurs, les mutuelles approuvées doivent accueillir des membres honoraires (notables, membres du clergé) qui apportent des ressources financières aux caisses en contrepartie de leur présence aux conseils d'administration. En 1856 alors que les mutuelles autorisées comptent en moyenne un membre honoraire pour 19 cotisants, le rapport est de 1 sur 4 pour les mutuelles approuvées. 
En contrepartie du contrôle politique les sociétés approuvées bénéficient de nombreux avantages : fiscalité plus légère, meilleurs taux pour les placements à la Caisse des dépôts et consignations, possibilités de recevoir des subventions des institutions publiques, autorisation de recevoir des dons et legs privés. Les mutuelles approuvées sont dotées des droits de personnes morales (possibilité de louer et d'acheter des biens mobiliers et immobiliers). Localement, les pouvoirs publics doivent fournir des locaux et autres fournitures pour faciliter l'activité des mutuelles. Ce cadre facilite grandement l'action des mutuelles approuvées, si bien qu'en 1870, $73 \%$ des mutuelles sont des mutuelles approuvées et elles regroupent deux tiers des mutualistes (ibid., p.25). Que les mutuelles soient approuvées ou autorisées, le " niveau élevé des droit d'entrée et cotisations [ne les mettent pas] à la portée de toute les bourses, on constate que les sociétés de secours mutuels s'adressent, en tout cas avant 1871, à une élite ouvrière. Ce sont des artisans, des ouvriers qualifiés que l'on retrouve dans les $\mathrm{SSM} \gg$ (Gueslin, 1999, p.8-9).

La période 1852-1869 connaît une forte croissance des sociétés approuvées qui passent de 2138 à 6139 et une baisse du nombre de sociétés autorisées de 2338 à 1741 (Gibaud, 1995). L'objectif de l'État est en partie réussi mais en partie seulement car la fraction la plus combative du mouvement ouvrier utilise le statut de mutuelle autorisée : chapeliers, tanneurs, tisseurs, etc. Ces mutuelles font l'objet d'une surveillance particulière, parce qu'il leur arrive de contourner la législation en se servant des cotisations en tant que caisse de grève. $\mathrm{Ce}$ contournement est bien connu des autorités et ne manque pas de justifier la critique de la politique impériale. En 1857 le procureur général de Lyon avertissait :

«Je sais que les sociétés de secours mutuels sont une création chérie du gouvernement. Mais les enfants préférés sont ceux qui ruinent les familles. On s'aveugle sur leurs défauts ; on se refuse à reconnaître leurs écarts, jusqu'au jour où il n'est plus temps d'y remédier. Il est très séduisant de penser qu'on peut amener le prolétaire à se secourir lui-même dans la maladie, dans la vieillesse ; il est très satisfaisant de croire que l'on échappera aux sociétés secrètes par les sociétés autorisées; il serait doux d'espérer que l'on formera une association immense, dévoué au gouvernement. Malheureusement tous ces résultats dérivés sont loin de la pensée de ceux qui acceptent les encouragements. Ils prennent l'arme qui leur est donnée ; mais ils entendent s'en servir à leur guise, et c'est au service de leur passion qui l'emploient. [...] Cette force a pour elle le nombre de ses soldats, leurs avidités, leurs espérances chimériques, certaine fausse conscience de leur droit, enfin ce courage, qui est vulgaire en ce pays et qui prend volontiers toutes les directions. Il ne leur manque absolument que l'organisation; et les prétendues sociétés de secours viennent la lui donner » (cité dans Cordillot, 1999, p.33, souligné par nous).

Si l'intention de l'État par le décret de 1852 est de détourner la classe ouvrière de la contestation en lui offrant une place dans l'Empire, la légalisation des mutuelles est en fait une arme contre l'ordre établi. Le désir d'auto-organisation, de démocratie, est une menace pour l'État et le capital. Il faut associer cette branche de la mutualité au mouvement coopérativiste qui se développe également au XIXème siècle et qui repose sur la volonté de dépasser le salariat (alors conçu comme soumission au capitaliste) par l'auto-organisation. Cette distinction est à la genèse de la séparation du mutuellisme et du syndicalisme (Gibaud, 1986, Dreyfus, 2001).

Comme le souligne Michel Cordillot (1999) la législation de l'Empire n'est pas le seul moteur de l'évolution des usages de la mutualité. Il faut chercher à l'intérieur du mouvement ouvrier la scission entre mutuellisme et syndicalisme. Le mouvement ouvrier était au début des 
années 1860 convaincu que la coopérative était le principal outil de transformation sociale. Mais, dans le feu des luttes, deux conceptions de la coopération se sont opposées : " ceux qui s'affirmaient partisans d'une confrontation sociale, y compris si elle devait être violente, et ceux qui réclamaient la solidarité générale de l'humanité [,] ceux qui voyaient la coopération comme le moyen devant permettre l'affranchissement du travail et ceux qui pensaient que la coopération, sous toutes ses formes, pouvait constituer une fin en soi » (ibid., p.35). Dans ce contexte, les sociétés de secours mutuels paraissent suspectes aux yeux des militants les plus véhéments qui y voient le moyen pour le patronat de s'exonérer de ses responsabilités. Les mutuelles étant par ailleurs construites sur une base territoriale et avec des membres honoraires (notables), elles ne permettent pas d'obtenir une homogénéité de classes entre leurs participants, ce qui nuit à la lutte contre l'exploitation.

L'ambivalence de la mutualité lui a valu d'être un espace pour le développement de la contestation ouvrière mais elle reste une institution soupçonnée de garantir l'ordre social. La fin des années 1860 montre les signes clairs de scission entre syndicalisme et mutuellisme. L'épisode de la Commune de Paris accélère la scission et préfigure la Sécurité sociale de 1945 (Taithe, 2003, Vahabi et al., 2020). En 1871, les parisiens luttent contre l'État républicain qui a choisi la défaite militaire face à la Prusse et la répression sociale. Dans leur résistance, les parisiens décident d'un programme social ambitieux sur des bases d'auto-organisation des travailleurs. Après avoir expérimenté durant le $\mathrm{XIX}^{\text {ème }}$ siècle de multiples formes d'autoorganisation comme les mutuelles et les coopératives, ils inventent La Sociale. C'est cette invention qui leur vaut la fureur de la III ${ }^{\mathrm{ème}}$ République se traduisant par la semaine sanglante.

\section{De la républicanisation à Vichy : l'intégration à l'ordre établi (1871-1945)}

Entre la chute de la Commune de Paris et le vote de la Charte de la Mutualité (1898), il s'écoule 27 ans. Le décret du 27 octobre 1870 adopté par le gouvernement provisoire abroge le principe de nomination des présidents des sociétés de secours mutuels par le pouvoir politique et supprime la Commission d'encadrement et de surveillance. L'élection du président redevient libre. Au début des années 1880, le mouvement syndicaliste, largement ouvrier, et le mouvement mutuelliste, ouvert à d'autres catégories sociales, se séparent. Cette évolution progressive favorise le rapprochement entre mutualité et République.

Lors du premier congrès de la mutualité, qui se tient à Lyon en 1883, la coupure avec le syndicalisme est acquise. 110000 sociétaires sont représentés pour 251 sociétés de secours mutuels. L'étude des invités semblent accréditer l'hypothèse d'un congrès organisé et destiné uniquement aux mutuelles approuvées. Si le règlement du congrès prévoit de situer les débats à un strict plan économique et de proscrire les positions politiques, Hippolyte Maze, député de l'Oise auteur du texte qui formera le socle de la Charte de la mutualité, brise l'interdit et pose clairement la mutualité comme une institution de stabilisation sociale contre toute volonté de bouleversement de l'ordre établi (Gibaud, 1986, p.49-50).

La Charte de la mutualité de 1898 entérine les principes de liberté d'adhésion et de solidarité entre cotisants (contre l'assurance) (Dreyfus et al., 1999). Elle inaugure l'âge d'or du mutualisme dont l'importance économique, politique et sociale ne cesse pas de croître jusqu'à la fin de la Seconde Guerre mondiale. En 1902, la loi permet la création de la Fédération nationale de la mutualité française (FMNF) qui restera pendant longtemps la voix politique de l'institution. La mutualité est de plus en plus perçue comme un point d'appui solide pour les républicains contre la radicalité du conflit capital-travail. Les élites républicaines (petite bourgeoisie, professions intellectuelles, etc.) investissent largement les mutuelles et pensent « que la mutualité peut aider à résoudre la question sociale » (Dreyfus, 2002, p.22). 
Le passage de la mutuelle d'Empire à la mutuelle républicaine peut s'interpréter comme le passage d'un paternalisme social à un autre, contre les aspiration d'auto-organisation du mouvement ouvrier. Cette tutelle, qu'elle soit impériale ou républicaine, n'est pas sans effet sur la forme que prend l'aide mutualiste. Le cas d'Henri Vermont, avocat chrétien, président de la société de secours mutuels l' «Émulation chrétienne de Rouen » permet d'illustrer les effets de la tutelle (Marec, 1999). L'Émulation chrétienne est une mutuelle créée en 1849, reconnue d'utilité publique en 1862 et dont le but est de réconcilier les différentes classes sociales. En 1871, au moment où Henri Vermont prend la direction de la société, celle-ci rencontre des problèmes financiers liés à la baisse du nombre de sociétaires et de membres honoraires. Il impose une politique de restriction des dépenses en repoussant l'âge de la retraite de 60 à 65 ans, en diminuant les pensions et en augmentant les cotisations. Cela vaut à l'Émulation d'être surnommée la «société du doigt dans l'œil » (ibid., cité p.51). Henri Vermont réussit à convaincre le patronat local de participer au financement de la société en leur expliquant que cela permet d'éviter le développement du socialisme. Une fois les finances à flot grâce aux membres honoraires, il lui est possible d'améliorer et de diversifier les prestations.

L'influence des notables dans les pratiques des mutuelles se donne aussi à voir dans le rapport à la santé - qui constitue l'axe principal de développement des mutuelles. Les mutuelles s'appuient sur une aspiration forte à la santé à la fin du XIXème siècle afin de moraliser les classes ouvrières (Dessertine et Faure, 1999, p.139). La volonté de moraliser la classe ouvrière par la santé implique une attention stricte quant à l'accès aux soins. L'un des grands enjeux est de distinguer les cas où le financement des soins par la mutualité est légitime ou non. Dès lors que la maladie est causée par l'intempérance, l'imprévoyance ou plus largement à l'inconséquence des ouvriers, alors l'accès aux soins est refusé. La littérature d'époque déborde de discussions que l'on pourrait rattacher aujourd'hui au concept d'aléa moral.

La violence de la conflictualité sociale à la fin du XIXème siècle contraint la IIIème République à réviser progressivement le rôle de l'État sur la question sociale. En 1893 la loi d'Assistance médicale gratuite offre un accès aux soins gratuits pour les plus pauvres. La charité se transforme en assistance publique mais cela reste insuffisant face aux enjeux. En 1898, la loi sur les accidents du travail impose l'obligation de cotisation mais sans intervention de l'État. Une première loi sur les retraites ouvrières et paysannes est votée en 1910, mais elle est peu appliquée, notamment en raison d'une décision du conseil d'État contre l'obligation de cotisation (1911).

L'enjeu de l'obligation de cotisation est particulièrement important tant pour l'État que pour la mutualité. L'État refuse l'obligation dans sa grande tradition du laissez-faire, laissez-passer. La mutualité refuse l'obligation en raison de l'un de ses principes cardinaux, la liberté de cotisation. La liberté est d'ailleurs l'une des raisons qui a poussé la mutualité à se séparer du courant syndicaliste moins hostile à l'obligation. Cependant, avec la loi sur les retraites ouvrières et paysannes d'une part et les lois d'assurances sociales d'autre part, la mutualité change radicalement de positionnement, voyant dans l'obligation de cotisation une source possible d'augmentation de son pouvoir et de son prestige. Devant le problème de l'obligation, la mutualité demande à assurer seule la gestion des caisses de retraite ouvrières et paysannes de 1910 et que des caisses mutualistes soient créées lorsqu'il n'en n'existe pas. Ce retournement conduit Jean Jaurès à remarquer ironiquement le double discours de la mutualité : «Voilà la mutualité qui devient à son tour obligatoire, administrative » (Gibaud, 1986, p.90).

La loi de 1910 ne donne pas satisfaction à la mutualité et consacre le principe de liberté d'affiliation : la cotisation est obligatoire mais le choix des caisses est libre. Ce cadre législatif 
explique la forte progression du mutualisme dont le nombre de cotisants passe de 1,9 millions en 1898 à 5,3 millions en 1914. Les effectifs ouvriers représentent une faible part du total du fait de l'impossibilité pour eux de cotiser (salaires faibles et précarité). La mutualité reste une institution destinée à l'élite ouvrière et surtout aux professions plus aisées (instituteurs, employés, etc.). Ce n'est qu'après-guerre que l'Assemblée vote les lois d'assurances sociales de 1928-1930. Ces lois, portant sur la santé et les retraites, suivent le même que celle de 1910. La liberté d'affiliation reste la règle mais, le principe d'obligation étant cette fois-ci définitif, le nombre de personnes concernées augmente considérablement et touche toutes les catégories de travailleurs. Contrairement à ce que l'on pourrait penser, la mutualité ne remporte pas l'essentiel des nouveaux cotisants. La loi crée des caisses départementales pour les cas où les cotisants n'avaient pas de caisse d'affinité (une préférence). Ce dispositif prévu comme un dispositif de secours est en fait devenu le cœur des lois de 1928-1930. Les caisses départementales gèrent les cotisations de 3,7 millions de cotisants, contre 1,6 millions pour la mutualité. Si la liberté d'affiliation n'a pas fonctionné au profit exclusif de la mutualité, celleci a vu son pouvoir grandir par un mécanisme d' "influence par le haut » (Gibaud, 1986, p.101). La loi prévoit en effet la présence forte des représentants de la mutualité dans les institutions régionales et nationales d'administration du nouveau système.

Pendant cette période d'âge d'or, la mutualité connaît une forte progression, atteignant 10 millions de sociétaires en 1938. Cette hausse n'est pourtant pas liée d'abord aux effets de la loi mais à celui des membres honoraires (+ $46 \%$ depuis 1920). La mutualité demeure une institution de notables de la IIIème République. Les lois d'assurances sociales lui confèrent une primauté dans la gestion des caisses malgré sa position non majoritaire en nombre d'affiliés. La mutualité fait ainsi valoir ses années d'expérience dans la gestion du social. Elle est devenue l'institution idéale pour l'État républicain qui craint autant l'intervention directe que l'autonomie ouvrière. Comme le souligne Pierre Laroque, la mutualité est progressivement devenue «l'instrument d'un nouveau type de paternalisme social » (Gibaud, 1986, p.11).

Après l'âge d'or, les années noires. Durant la Seconde Guerre mondiale, les responsables de la FNMF choisissent de suivre Vichy plutôt que la résistance (Souchet, 2001). Ils s'accommodent parfaitement des aspirations corporatistes du régime de Vichy. Le principe de neutralité qui avait servi à choisir les élites de la IIIème République plutôt que la branche syndicaliste du mouvement ouvrier refait surface et conduit à préférer la Charte du travail à la résistance. L'objet de la Charte du travail n'est-elle pas, comme celui de la mutualité, de nier toute différence de classe de façon à pacifier les relations entre patrons et ouvriers ? En 1942, la FNMF fait paraître sa doctrine sur Vichy dans La mutualité et la Charte du travail où elle assume pleinement leur convergence.

Tout l'enjeu sur la position de la mutualité par rapport à Vichy est de délier ce qui relève de la convergence réelle et ce qui relève de la contrainte. Selon Bernard Gibaud, la mutualité serait tombée dans «les pièges du neutralisme » (1986, p.104). Néanmoins, on peut aussi souligner la continuité stratégique de l'institution qui, depuis sa séparation d'avec le mouvement ouvrier combatif, a systématiquement préféré l'ordre établi sa critique. D'embryon de la démocratie sociale, la mutualité s'est de plus en plus affirmée comme une institution des classes moyennes favorables à la négociation avec l'État et le capital. C'est ce qui la conduit à accepter l'obligation de cotisation (et même à demander l'obligation d'affiliation) contre son principe cardinal de liberté. On peut alors se demander si le devenir vichyste de l'institution ne relève que de l'erreur de parcours ? 


\section{La mutualité traditionnelle contre la Sécurité sociale (1945-1947)}

Le 15 mars 1944, le Conseil national de la Résistance adopte son programme pour la France libérée qui prévoit «un plan complet de Sécurité sociale ». La Sécurité sociale de 1945 constitue-t-elle une rupture ou une continuité avec les institutions du passé ?

Selon Brunot Valat (2001, 2006), il est incontestable que l'institution marque un changement quantitatif par rapport aux assurances sociales de 1928-1930. Au $1^{\text {er }}$ juillet 1946 les assurances sociales sont remplacées par le régime général de Sécurité sociale. Celui-ci concerne 20 millions de bénéficiaires au lieu de 14 millions précédemment, le montant des prestations est revalorisé et les conditions d'ouverture sont élargies (60 heures de travail au lieu de 60 jours). Au registre de l'amélioration des prestations, les suivantes sont particulièrement notables: suppression du plafonnement des indemnités journalières, réduction du délai de carence de 5 à 3 jours, suppression de la limitation du remboursement des médicaments, suppression du ticket modérateur en chirurgie (où les abus sont moins probables), remboursement à $80 \%$ des soins médicaux, création de l'assurance longue maladie (passage de 6 mois à 3 ans). Ceci engendre une hausse des dépenses de santé qui impose l'augmentation du taux de cotisation (de $4 \%$ en 1944 à $6 \%$ des salaires en 1948). En dépit, de ces améliorations, Bruno Valat soutient l'existence d'une continuité forte avec les assurances sociales de 1928-1930. Le grand principe de gestion par des caisses ne varie pas et les nouveautés s'appuient sur l'existant.

Il paraît évident que la Sécurité sociale de 1945 n'est pas une institution entièrement neuve. Néanmoins, on peut suivre Bernard Friot lorsqu'il explique que 1945 constitue une rupture dans l'histoire du mouvement ouvrier et des assurances sociales (Friot, 2017). Selon lui, la radicalité de 1945 n'est pas la Sécurité sociale dont toutes les institutions sont effectivement présentes avant-guerre, mais le régime général de Sécurité sociale. Le régime général de Sécurité sociale (qui concerne les travailleurs salariés du commerce et de l'industrie) est une discontinuité fondamentale sur trois plans :

- L'unité des caisses : contre la division des caisses d'avant-guerre, le régime général regroupe dans une seule caisse les différentes catégories de risque. Cela va à l'encontre de la liberté d'affiliation prévue par les lois de 1928-1930. Ce principe permet de donner une assise financière plus forte à l'institution.

- Le taux de cotisation interprofessionnel unique : contrairement à l'avant-guerre, il n'existe qu'un seul taux de cotisation, ce qui permet d'éviter la concurrence sur les taux entre différentes branches ou entre différents employeurs.

- La gestion par les intéressés : le pouvoir de décision dans les comités d'administration est confié aux intéressés eux-mêmes qui élisent leurs représentants. Les salariés reçoivent $3 / 4$ des sièges et $1 / 4$ est attribué au patronat.

La gestion par les intéressés constitue une rupture fondamentale avec les institutions d'avantguerre. Après la Commune de Paris, la mutualité est devenue progressivement une institution dirigée par les notables de la III ${ }^{\text {ème }}$ République. En ce sens, le régime général de Sécurité sociale renoue avec une autre origine de la mutualité, celle de la subversion de l'ordre établi au nom de la démocratie sociale et politique. La Sécurité sociale de 1945 ne concerne pas uniquement une élite ouvrière en capacité de payer les cotisations, mais tous les ouvriers cotisants qui détiennent par ailleurs le contrôle politique de l'institution. À une époque marquée par la résistance face au capital et à l'État, la Sécurité sociale est un pas majeur vers la démocratisation. Ce mouvement vers la démocratisation se fait contre la mutualité républicaine et vichyste mais aussi contre les autres formes de tutelles que sont les caisses à initiative patronale ou cléricale. 
Il faut bien sûr se garder d'une présentation caricaturale de la Sécurité sociale de 1945 : la rupture n'est pas complète et l'on n'observe pas l'avènement d'une institution parfaitement démocratique et indépendante de l'État et du capital. L'article 25 de l'ordonnance du 4 octobre 1945 précise que l'État a le pouvoir d'annuler les décisions de la Sécurité sociale si elle lui semble «contraire à la loi ou de nature à compromettre l'équilibre financier de la caisse ». La Sécurité sociale ne dispose pas d'autonomie financière : le niveau des cotisations et des prestations est soit décidé soit étroitement contrôlé par l'État au nom de l'équilibre budgétaire. L'État peut « empêcher d'agir mais non contraindre à agir » (Dreyfus et al. 2006). L'étau de l'État se resserre d'ailleurs très rapidement avec la mise en place dès 1949 d'un contrôle a posteriori des comptes de la Sécurité sociale par la Cour des comptes. Bien d'autres limites aux principes du régime général peuvent être mentionnées (Friot, 2017) : séparation des caisses de Sécurité sociale et d'allocations familiales, création de caisses d'indépendants, d'agriculteurs, de cadres, etc., plafonnement des cotisations en fonction des salaires, réduction du nombre de voies aux salariés dans les caisses d'allocations familiales, etc.

En 1945 de nombreux obstacles se dressent face à la démocratisation de la Sécurité sociale. Néanmoins, on ne peut mesurer la rupture qu'à l'aune de l'histoire longue du mouvement ouvrier et mutualiste. Depuis 1789, les classes populaires urbaines et rurales sont tenues à distance du pouvoir politique et économique. Elles ont alors dû lutter simultanément contre le capital et l'État - ni l'un ni l'autre n'étant prêt à les admettre comme entité politique à part entière. Elles ont créé des institutions de contestation auto-organisées face à l'ordre établi, les coopératives de production mais aussi les sociétés de secours mutuels. Face à ce danger de remise en cause de l'ordre établi, l'État a mis en place un cadre législatif et financier permettant une réappropriation de l'institution, sous le Second Empire mais aussi sous la IIIème République. La Sécurité sociale est un symbole de conquête du pouvoir par les classes populaires. Une conquête limitée, mais néanmoins significative.

Cette conquête n'est pas du goût de tous et la Sécurité sociale est combattue dès sa création notamment par les médecins, les employeurs, le clergé, la droite politique et une partie de la SFIO. La position de la mutualité est ambivalente. D'un côté, la FNMF s'est dressée sans réserve contre l'institution. Lors de son assemblée générale du 5 mai 1945 elle reproche notamment à la Sécurité sociale d'être le fruit d'un pouvoir législatif irrégulièrement constitué et de mener une étatisation de la protection (Gibaud, 1986, p.129). Selon Gaston Tessier, syndicaliste CFTC membre du conseil d'administration de la FNMF, la Sécurité sociale n'est rien d'autre qu'une menace totalitariste (ibid., p.132). La FNMF représente essentiellement la position des mutuelles traditionnelles (liées aux professions indépendantes) ce qui est en décalage croissant avec la progression des salariés dans les effectifs mutualistes Ainsi, d'un autre côté, certains secteurs de la mutualité ne s'opposent pas systématiquement à la Sécurité sociale. C'est le cas par exemple des mutuelles de fonctionnaires qui se regroupent en 1946. La progression des mutuelles professionnelles et d'entreprise après 1945 implique un rapprochement avec le syndicalisme qui participe aussi au soutien à la Sécurité sociale. Dans ce contexte, la FNMF essaie de trouver un espace pour sa survie et négocie avec la Fédération nationale des organismes de Sécurité sociale (créée le 24 juin 1946) un accord qui servira de base à la loi Morice de mars 1947. Cette loi autorise la mutualité à constituer des sections locales, ce qui lui donne une position d'organisme complémentaire. En outre, la loi d'avril 1947 offre à la mutualité la gestion du régime obligatoire des de fonctionnaires (Siney-Lange, 2015). L’avenir de la mutualité est assuré.

\section{Conclusion}


Ce parcours dans l'histoire économique de la mutualité a mis en évidence deux périodes cohérentes. Une première période entre la Révolution française et 1871 voit se développer une mutualité ambivalente, entre la contestation de l'ordre capitaliste et son intégration. La seconde période de la mutualité commence après 1871 lorsque la tentative de réappropriation par le Second Empire (décret de 1852) porte ses fruits. Elle n'est plus le lieu de la subversion de l'ordre établi, elle s'institutionnalise et accepte le rôle de modérateur du capitalisme. Alors qu'elle se présente comme indépendante face au politique, cette mutualité doit sa splendeur à l'État. Il n'est pas étonnant qu'elle devienne une force de la conservation et qu'elle s'oppose en 1945 à la Sécurité sociale. La Sécurité sociale de 1945 renoue avec l'ambition d'autoorganisation et de démocratie sociale, ce qui est devenu insupportable aux yeux de la mutualité des notables.

$\mathrm{Au}$ total, il est possible alors de reformuler la position de Michel Dreyfus selon qui «[1]'instauration de la Sécurité sociale à la Libération représente une revanche des syndicalistes sur les mutualistes »(2002, p.25). Ce jugement pose l'implicite d'une homogénéité de la mutualité et du syndicalisme. Or il y a bien deux périodes et deux formes de la mutualité. L'instauration de la Sécurité sociale à la Libération représente la revanche d'une forme de la mutualité sur une autre, la revanche de la mutualité subversive sur la mutualité conservatrice.

En quoi cette histoire peut-elle nourrir la réflexion sur les enjeux contemporains à propos du partage de territoire entre Sécurité sociale et mutualité ? Comme la mutualité avant elle, la Sécurité sociale est passée progressivement sous l'influence de l'État qui contrôle désormais l'institution aux dépens des intéressés (Batifoulier et al., 2020, Palier, 2005). La Sécurité sociale est aujourd'hui dirigée par les " élites du welfare » comme la mutualité d'avant-guerre était dirigée par les notables républicains. D'une certaine manière, la lutte de la mutualité contre la Sécurité sociale de 1945 a porté ses fruits. L'enjeu principal du partage entre Sécurité sociale et mutualité est devenu technique : quelle est la modalité d'assurance des soins la plus efficace ? Cette façon de poser le débat est problématique dans la mesure où elle oublie que l'originalité de la mutualité et de la Sécurité sociale est qu'elles ont partie liée avec la lutte des classes : contre le capital et l'État, pour l'extension des droits démocratiques.

\section{Bibliographie}

Batifoulier P., Da Silva N. et Duchesne V., 2019, « The Dynamics of Conventions: The Case of the French Social Security System », Historical Social Research, Vol.44(167), p.258-284.

Batifoulier P., Da Silva N., et Vahabi M., 2020, «La Sociale contre l'État providence. Prédation et protection sociale », Document de travail CEPN, HAL, en ligne.

Cordillot M., 1999, « Le mouvement ouvrier français à la croisée des chemins : mutualisme et/ou résistance à la fin du Second Empire », dans Dreyfus et al. (dir) : Démocratie, solidarité et mutualité : autour de la loi de 1898, Paris, Mutualité française, p.27-39.

Daviet J-P., 1999, «Un rêve de liberté et de bonheur. De la loi Le Chapelier aux associations ouvrières et à la mutualité », dans Dreyfus et al. (dir) : Démocratie, solidarité et mutualité : autour de la loi de 1898, Paris, Mutualité française, p.40-48.

Dessertine D. et Faure O., 1999, «La mutualité et la médicalisation de la société française (1880-1980) », dans Dreyfus et al. (dir) : Démocratie, solidarité et mutualité : autour de la loi de 1898, Paris, Mutualité française, p.138-149. 
Didry C., 2016, L'institution du travail. Droit et salariat dans l'histoire, Paris, La Dispute.

Dreyfus M., 2001, Liberté, égalité, mutualité : mutualisme et syndicalisme, 1852-1967, Paris, Éditions de l'Atelier.

Dreyfus M., 2002, «Bonnes feuilles Liberté, Égalité, mutualité. Mutualisme et syndicalisme (1852-1967), Éditions de l'Atelier, $2001 »$, Mouvements, Vol.1(19), p.21-28.

Dreyfus M., Gibaud B. et Gueslin A., 1999, Démocratie, solidarité et mutualité. Autour de la loi de 1898, Paris, Economica.

Dreyfus M., Voldman D., Ruffat M. et Viet V., 2006, Se protéger, être protégé : une histoire des assurances sociales en France, Rennes, PUR.

Friot B., 2017, Vaincre Macron, Paris, La Dispute.

Gibaud B., 1986, De la mutualité à la Sécurité sociale. Conflits et convergences, Paris, Les éditions ouvrières.

Gibaud B., 1995, «Mutualité/Sécurité sociale : un couple sous tension », Vingtième siècle, revue d'histoire, (48), p.119-130.

Gueslin A., 1999, «Introduction à la première partie. L'affirmation d'une pratique sociale singulière », dans Dreyfus et al. (dir) : Démocratie, solidarité et mutualité : autour de la loi de 1898, Paris, Mutualité française, p.3-11.

Hatzfeld H., 1971, Du paupérisme à la Sécurité sociale, (1850-1945), Paris, Armand Colin.

Hobsbawm E., 1988, L’ère des révolution, Paris, Éditions complexes.

Kates K., 2006 (Ed.), The French Revolution, recent debates and new controversies, second edition, New York, Routledge.

Marec Y., 1999, « Un homme d'œuvre et la Mutualité. Henri Vermont (1836-1928)», dans Dreyfus et al. (dir) : Démocratie, solidarité et mutualité : autour de la loi de 1898, Paris, Mutualité française, p.50- 63.

Margairaz D., 1991, «Le maximum : une grande illusion libérale ou la vanité des politiques économiques », État, finances et économie pendant la Révolution française, Comité pour l'histoire économique et financière de la France, Paris, p.399-428.

Palier B., 2005, Gouverner la Sécurité sociale : les réformes du système français de protection sociale depuis 1945, Paris, Presses universitaires de France.

Skocpol T., 1979, States and Social revolutions in France, Russia, and China, New York, Cambridge University Press.

Siney-Lange C., 2015, À l'initiative sociale. Les grands combats de la MGEN, Paris, Presses du Châtelet.

Taithe B., 2003, Citizenship and Wars: France in Turmoil 1870-1871, London, Routledge.

Toucas-Truyen P., 1999, «Les sociétés approuvées du Second Empire », dans Dreyfus et al. (dir) : Démocratie, solidarité et mutualité : autour de la loi de 1898, Paris, Mutualité française, p.14-25.

Vahabi M., Batifoulier P. et Da Silva N., 2020, «A theory of predatory welfare state and citizen welfare: the French case », Public Choice, Vol.182(3-4), p.243-271. 
Valat B., 2001, Histoire de la Sécurité sociale (1945-1967) : l'État, l'institution et la santé, Paris, Economica.

Valat B., 2006, «Le choix de la Sécurité sociale (1944-1948)», dans Se protéger, être protégé, Dreyfus et al. (dir), Rennes, PUR, p.259-334.

Weintrob L., 1999, « L'apprentissage de la solidarité républicaine. Les SSM à Lyon (18041914) », dans Dreyfus et al. (dir) : Démocratie, solidarité et mutualité : autour de la loi de 1898, Paris, Mutualité française, p.89-99. 